\title{
Extremal periodic wave profiles
}

\author{
E. van Groesen ${ }^{1,2}$ and Andonowati ${ }^{1,2,3}$ \\ ${ }^{1}$ Department of Applied Mathematics, University of Twente, The Netherlands \\ ${ }^{2}$ LabMath-Indonesia, Bandung, Indonesia \\ ${ }^{3}$ Department of Mathematics, Institut Teknologi Bandung, Indonesia
}

Received: 6 October 2006 - Revised: 5 December 2006 - Accepted: 5 December 2006 - Published: 15 January 2007

\begin{abstract}
As a contribution to deterministic investigations into extreme fluid surface waves, in this paper wave profiles of prescribed period that have maximal crest height will be investigated. As constraints the values of the momentum and energy integrals are used in a simplified description with the $\mathrm{KdV}$ model. The result is that at the boundary of the feasible region in the momentum-energy plane, the only possible profiles are the well known cnoidal wave profiles. Inside the feasible region the extremal profiles of maximal crest height are "cornered" cnoidal profiles: cnoidal profiles of larger period, cut-off and periodically continued with the prescribed period so that at the maximal crest height a corner results.
\end{abstract}

\section{Introduction}

This paper deals with uni-directional spatially periodic waves of maximal crest height. The maximization property is specified by describing the constraints that should be satisfied. As constraints we take the momentum integral $M$ and the Hamiltonian integral $H$ (the energy) since these are dynamically invariant integrals for conservative (Hamiltonian) wave equations with translation symmetry. This research is a continuation of a recent paper on finite energy solutions of maximal crest height, Van Groesen and Andonowati (2006a), and contributes to the investigation of mathematical-physical properties of "extreme waves", also called rogue or freak waves. The extremal wave profiles we obtain will provide upper bounds on the maximal wave crest that can be obtained, depending on the initial data (which specify the values of momentum and energy).

Such mathematical-physical investigations are different from complementary interest in statistical properties of rogue waves that determine the probability of occurrence of waves

Correspondence to: E. van Groesen

(groesen@math.utwente.nl) of a specified crest (or wave) height, which height may be arbitrarily large; see for instance recent proceeding reports and references therein for contributions in both areas Olagnon and Prevosto (2004); Rogue Waves (2005). In the following we will first consider a simple linear problem which results when the constraint integrals are quadratic; this can be seen as approximating the Hamiltonian by a quadratic expression as is custom for small amplitude waves described by linear dispersive wave equations. Then we will investigate waves described by the Korteweg-de Vries equation. In each case we find a special curve in the parameter space $(m, h)$ of values of the constraints $M, H$. For points on this curve, for which $h=\mathcal{H}(m)$, the (only possible) profiles of maximal crest height are smooth, and given by harmonic and by $\mathrm{KdV}$ cnoidal wave profiles respectively. This curve is the boundary of the feasible region; for points above this curve, $(m, h)$ with $h>\mathcal{H}(m)$, the periodic profiles of extremal crest height are non-smooth: they are cornered profiles which consist of parts of harmonic or catenray profiles and of cnoidal profiles, respectively, that meet at an angle at the point of highest crest.

The smooth harmonic and cnoidal profiles evolve under the dynamics in a special way as Hamiltonian relative equilibria: pure translations at constant speed. The dynamics of the cornered profiles is more complicated; although dispersion will initially smoothen the corner, (near-) recurrence cannot be excluded.

In Sect. 2 we briefly describe the general methodology to formulate and describe profiles of extremal periodic waves, and summarise the KdV model. In Sect. 3 we consider the linearised model by neglecting cubic contributions in the Hamiltonian; in Sect. 4 for the nonlinear KdV model the cornered cnoidal waves are presented. Section 5 provides conclusions and some comments.

Published by Copernicus GmbH on behalf of the European Geosciences Union. 


\section{Methodology}

We denote by $\eta(x, t)$ real valued wave fields depending on the spatial and temporal variables $x, t$; since we are mainly concerned with the profiles we will simply write $\eta(x)$. The interest will be in uni-directional spatially periodic solutions, with period $L$, that are governed by a Hamiltonian system with Hamiltonian $H$ and a momentum integral $M$. For definiteness we take as governing evolution equation the Hamiltonian system that describes waves running mainly in one direction

$\partial_{t} \eta=-\partial_{x} \delta H(\eta)$.

Here the Hamiltonian $H$ is a translation invariant integral which is linearly independent from the momentum functional $M=\int \frac{1}{2} \eta^{2} d x$

which itself is an integral of the motion, with translation as its Hamiltonian flow.

\subsection{Extremal crest formulation}

Denote the maximal crest height functional for functions $\eta$ by

$$
C(\eta):=\max _{x} \eta(x) .
$$

Then the extremal problem can be written as

$$
\max _{\eta}\{C(\eta) \mid \eta \in \mathcal{C}\}
$$

where the constraint set $\mathcal{C}=\mathcal{C}(h, m)$ consists of functions $\eta$ that are $L$-periodic and satisfy

$A(\eta)=0, H(\eta)=h, M(\eta)=m$.

Here the average is defined as $A(\eta)=\int_{0}^{L} \eta(x) d x$. By defining the value function as the extremal crest height for given constraint values $(m, h)$ :

$V(h, m):=\max _{\eta}\{C(\eta) \mid \eta \in \mathcal{C}(h, m)\}$,

we get from the time invariance of $M$ and $H$ the dynamic result that at each position and time the evolution from an initial value $\eta_{0}(x)$ will be such that

$\eta(x, t) \leq V\left(m_{0}, h_{0}\right)$ where $m_{0}=M\left(\eta_{0}\right), h_{0}=H\left(\eta_{0}\right)$.

For the maximal rest formulation to be sensibly defined, it is necessary that the constraint set is non-empty. This will depend very much on the energy and momentum functional. In the KdV model that we will consider in this paper, the Hamiltonian will be bounded above for given value of momentum. (The reduced Hamiltonian to be defined later will be bounded below.) That means that then the constraint set is nonempty only if the values $(m, h)$ are "feasible" by satisfying certain conditions, namely that $h$ is not larger than its maximal value for given $m$.

For the KdV model we can nicely define the boundary of the feasibility set, as is described in the next subsection.

\subsection{Relative Equilibrium solutions}

The boundary in the parameter space of the feasible set is given by a curve that can be obtained from a separate extremal formulation. Namely, by the extremizers of the Hamiltonian on level sets of the momentum. If we define this optimization problem ${ }^{1}$ and its value function by

$\mathcal{H}(m):=\max _{\eta}\{H(\eta) \mid A(\eta)=0, M(\eta) \equiv m\}$,

the condition of feasibility is that the pair $(m, h)$ satisfies $h \leq$ $\mathcal{H}(m)$, which specifies in the $M, H$-plane the points below the graph of the value function $m \rightarrow \mathcal{H}(m)$.

This last variational problem is special for Hamiltonian systems; the extremals are profiles for which the Hamiltonian $H$-dynamics is the same as the Hamiltonian $M$-dynamics (up to some scaling in time), which means a pure translation when $M$ is momentum. Such solutions are known in Classical Mechanics as "Relative Equilibria" (RE) solutions; see Van Groesen and De Jager (1994) for the generalizations to wave problems. For instance, for $\mathrm{KdV}$ the resulting solutions are the well known cnoidal-wave solutions, as we will see in Sect. 2.5 and Sect. 4.

For this reason we will call the parameter curve $(m, \mathcal{H}(m))$ the RE-curve.

In more detail, a RE profile satisfies the Lagrange multiplier rule (LMR): for some multipliers

$\lambda_{H} \delta H(\eta)+\lambda_{M} \delta M+\mu=0$.

This can be simplified somewhat when the constraint set is not singular, which means that $m$ is such that $\lambda_{M} \delta M+\mu=0$ only if $\lambda_{M}=\mu=0$. In that case we have that $\lambda_{H} \neq 0$, and therefore we can normalise its value to be -1 without restriction. Then we get as equation:

$\delta H(\eta)=\lambda_{M} \delta M+\mu$.

In the next subsection we will show that these RE are the profiles of maximal crest height for parameter values $(m, h)$ on the RE-curve.

\subsection{Cornered RE profiles}

Now we consider the extremal problem of interest:

$\max _{\eta}\{C(\eta) \mid \eta \in \mathcal{C}(h, m)\}$.

In order to write down the governing equation, we need the variational derivative of the crest height functional $C(\eta)$ : $=\max _{x} \eta(x)$. Assuming that the maximal value is attained at isolated points, the variational derivative is given by Dirac's

\footnotetext{
${ }^{1}$ We here assume that the momentum is coercive, in functional analytic sense, with respect to the Hamiltonian. Roughly speaking this means that the maximization problem is well-posed: there is a finite largest value that is attained for certain maximizers, while the minimization problem is ill-posed.
} 
delta function centered at the position of maximal crest height, say at $x_{\max }$

$\delta C(\eta)=\delta_{\text {Dirac }}\left(x-x_{\max }\right)$.

Then Lagrange's multiplier rule leads to the equation

$\sigma \delta_{\text {Dirac }}\left(x-x_{\max }\right)=\lambda_{H} \delta H(\eta)+\lambda_{M} \delta M(\eta)+\mu$.

It is to be noted that when $\sigma=0$, we arrive at the equation of RE treated above. Indeed, we can distinguish two cases, depending on whether the constraint set is singular or not. We briefly recall the basic definitions here.

A "singular point" of the constraint set is a point $\bar{\eta}$ for which the functionals are dependent, i.e. for which $\lambda_{H} \delta H(\bar{\eta})+\lambda_{M} \delta M(\bar{\eta})+\mu=0$ for some non vanishing vector $\left(\lambda_{H}, \lambda_{M}, \mu\right)$. Such a point satisfies the above multiplier equation for $\sigma=0$. In that case the constraint set is called singular, and the specific values $(m, h)$ are called singular values. If there are no singular points, the constraint set is called regular.

Hence, with the description above, we get that the boundary of the feasible region is the RE-curve, and the extremal profiles are the RE. Since these RE are the only functions for these parameters on the RE-curve, these RE profiles have extremal crest height because there are no competing functions to be considered.

On the other hand, for a regular constraint set, a critical point will satisfy the LMR for some nonzero $\sigma$, which can then be normalised to one, $\sigma=1$, and we have

$\delta_{\text {Dirac }}\left(x-x_{\max }\right)=\lambda_{H} \delta H(\eta)+\lambda_{M} \delta M(\eta)+\mu$.

The effect of the appearance of the Dirac delta function will be that the optimal profile has a corner at the extremal position $x_{\max }$. But we can conclude even more from the local character of the delta function: outside the extremal position, the profile satisfies the equation satisfied by RE profiles. Therefore, a cornered solution will consist of a suitable composition of parts of RE-profiles (with different values of momentum $m$ than the prescribed value).

\subsection{Summary}

Although various technical aspects have to be verified and specialised, the above general reasoning leads to the following conclusions about extremal wave profiles in the setting we have sketched above.

Proposition. For an autonomous Hamiltonian wave equation with momentum conservation, the RE profiles are the only profiles (and hence have maximal crest height) for values of Hamiltonian and momentum that are on the RE-curve. For feasible values of Hamiltonian and momentum outside the RE-curve, cornered RE profiles are the maximizing profiles.
Remark. The results can in principle be extended to other cases for which there are other, or more, integrals in involution. However, the two integrals that we use, the Hamiltonian (energy) and the momentum, seem to be the most relevant ones to consider, since they are the basic integrals which exist in any realistic model of surface gravity waves above a horizontal bottom.

\subsection{Korteweg - de Vries model}

In the rest of this paper we will illustrate the methodology for the case of surface water waves on a layer of depth $D$ above a flat bottom, in the approximation of rather long, rather small amplitude waves. Unidirectional waves are then well described by the Korteweg - de Vries (KdV) type of equations, which are of the form (1).

Taking exact dispersion properties, but simplified nonlinearity, would provide the Hamiltonian for surface waves

$H=\int\left[\frac{1}{2} \eta C_{p} \eta+\frac{c_{0}}{4 D} \eta^{3}\right] d x$,

where the pseudo-differential operator $C_{p}$ has as symbol the phase velocity of dispersive infinitesimal waves on a layer of depth $D$ :

$c_{p}=c_{0} \sqrt{\tanh (k D) /(k D)}$ with $c_{0}=\sqrt{g D}$.

Here $c_{0}$ is the (largest) speed of long waves, and $g$ is the gravitational acceleration. The first term in the integrand of the Hamiltonian produces the linear part of the equation and describes the dispersion for small waves; the cubic term in the integrand provides the lowest order nonlinear effects. The governing equation reads

$\partial_{t} \eta=-\partial_{x}\left[C_{p} \eta+\frac{3 c_{0}}{4 D} \eta^{2}\right]$.

To simplify the description somewhat, we write the Hamiltonian as a deviation from a multiple of the momentum functional by introducing the reduced Hamiltonian as follows

$H=c_{0} M-c_{0} H_{r e d}$

with

$H_{\mathrm{red}}=\int\left[\frac{1}{2} \eta\left[1-C_{p} / c_{0}\right] \eta-\frac{1}{4 D} \eta^{3}\right] d x$.

The extremal formulation for the relative equilibria for $H$ and these of $H_{\text {red }}$ are similar, except that the constrained maximization of $H$ turns into a constrained minimization problem for $H_{\text {red }}$ :

$$
\begin{aligned}
& \max _{\eta}\{H \mid M=m, A(\eta)=0\} \\
& =c_{0} m-c_{0} \min _{\eta}\left\{H_{\text {red }} \mid M=m, A(\eta)=0\right\}
\end{aligned}
$$


The corresponding multipliers that determine the translation speed of the relative equilibrium solutions are related, according to

$\delta H=\lambda_{M} \delta M+\mu$

and

$\delta H_{\text {red }}=\lambda_{\text {red }} \delta M+\mu_{\text {red }}$

with

$\lambda_{M}=c_{0}\left(1-\lambda_{\text {red }}\right), \mu=-c_{0} \mu_{\text {red }}$.

The result for the multiplier $\lambda_{M}$ indicates that $H_{\text {red }}$ essentially describes the waves in a frame moving with the speed $c_{0}$.

To simplify matters, and to arrive at the classical $\mathrm{KdV}$ equation, we now approximate the pseudo-differential operator by its lowest order differential operator, leading to the approximation for $H$ by the Hamiltonian $H_{\mathrm{KdV}}$ :

$H \approx H_{\mathrm{KdV}}=c_{0} \int\left[\frac{1}{2} \eta^{2}-\frac{D^{2}}{12}\left(\partial_{x} \eta\right)^{2}+\frac{1}{4 D} \eta^{3}\right] d x$

The governing equation reads

$\partial_{t} \eta=-c_{0} \partial_{x}\left[\eta+\frac{D^{2}}{6} \partial_{x}^{2} \eta+\frac{3}{4 D} \eta^{2}\right]$.

Apart from a convenient scaling, we will consider the maximal crest problem for $H_{\mathrm{KdV}}$ in Sect. 4. For later reference we note that waves that travel undisturbed at speed $V$ are of the form $\eta(x-V t)$ and satisfy the equation

$\mu_{1}+V \eta=c_{0}\left[\eta+\frac{D^{2}}{6} \partial_{x}^{2} \eta+\frac{3}{4 D} \eta^{2}\right]$

This is precisely the equation for relative equilibria (3), with $\lambda_{M}=V$; periodic solutions are the cnoidal wave profiles that we will investigate in detail in Sect. 4.

To simplify the formulas somewhat, we scale the spatial variable and the wave height with the water dept, $\tilde{x}=x / D, \tilde{\eta}=9 \eta /(2 D)$, leading to the normalised equation

$-\partial_{\tilde{x}}^{2} \tilde{\eta}-\tilde{\eta}^{2}=\lambda \tilde{\eta}+\mu$

with $\mu=-27 \mu_{1} /\left(D c_{0}\right)$ and

$\lambda=6\left(c_{0}-V\right) / c_{0}$.

The above equation will be investigated in the following; the corresponding Hamiltonian is the scaled version of the deviation $H_{\text {red }}$ and is given by (omitting the tildes)

$H=\int\left[\frac{1}{2}\left(\partial_{x} \eta\right)^{2}-\frac{1}{3} \eta^{3}\right] d x$.

This will be the Hamiltonian we will consider in the rest of the paper. In the next section we will first consider the linear problem, neglecting the cubic term in this Hamitonian; in Sect. 4 we will investigate the full problem.

\section{Harmonics and cornered harmonic profiles}

We now investigate the case of a quadratic Hamiltonian. This corresponds to an approximation of the energy for systems that are linearised. This may serve as an approximation of more realistic nonlinear Hamiltonian systems such as the one above and in the next section. Any definite quadratic functional could be taken, but we will take the lowest order correction to surface gravity dispersion

$H(\eta)=\int\left(\frac{1}{2} \partial_{x} \eta\right)^{2} d x$.

The Eq. (4) for the extremal profiles becomes

$\sigma \delta_{\text {Dirac }}\left(x-x_{\max }\right)=\left(\lambda_{M}-\lambda_{H} \partial_{x}^{2}\right) \eta+\mu$.

To simplify the formulas somewhat, we take the length of the basic interval $L=2 \pi$. According to the Poincare-Friedrichs inequality, the value $h$ has to satisfy $h \geq \mathcal{H}(m)$ with $\mathcal{H}(m)$ : $=m$ in order that the values $(m, h)$ are feasible, i.e. in order that the constraint set is nonempty. Actually, because of the homogeneity of the functionals, it is possible to choose $m=1$. The constraint set is singular for $h=\mathcal{H}(m)$ and then the solutions are simply the harmonic functions that can be smoothly continued periodically. In detail, for $h=\mathcal{H}(m):=m$ we get

$\sigma=\mu=0, \lambda_{M}=1, \lambda_{H}=-1, \bar{\eta}(x)=\sqrt{\frac{m}{2 \pi}} \cos (x+\phi)$

These profiles are the RE profiles for this case: they are the solutions of the optimization problem

$\bar{\eta} \in \min _{\eta}\{H(\eta) \mid A(\eta)=0, M(\eta)=m\}=\mathcal{H}(m)$.

This is precisely the formulation of the eigenvalue problem related to the functionals $H$ and $M$. The RE curve is here the straight line $h=\mathcal{H}(m)$. The constraint set is regular for all values of $h>\mathcal{H}(m)$. Then the profiles of the optimal solutions will have corners. These profiles are of trigonometric or hyperbolic shape, depending on the value of $h / \mathrm{m}$. Since they can be shifted over an arbitrary distance, we choose to take the origin in the through, and let the corner appear at $x= \pm \pi$.

The solutions on the trigonometric branch exist for $\mathcal{H}(m)<h<h_{c r}$ where $h_{c r}=\left(15 / \pi^{2}\right) \mathcal{H}(m)$. Introducing a parameter $q$, these solutions are on the interval $[-\pi, \pi]$ given for $-1<q<0$ and $a>0$ by

$\eta_{\text {tri }}=a\left[\frac{1}{q \pi} \sin (q \pi)-\cos (q x)\right]$

Solutions on the so-called "catenary ${ }^{2}$ branch" are defined for $h>h_{c r}$ with profiles given on the interval $[-\pi, \pi]$ for any $q>0$ and for $A>0$ by

$\eta_{\mathrm{cat}}=A\left[-\frac{1}{q \pi} \sinh (q \pi)+\cosh (q x)\right]$.

\footnotetext{
${ }^{2}$ The name catenary is used since these solutions have similar form than the shape of a hanging chain which has cosh description.
} 


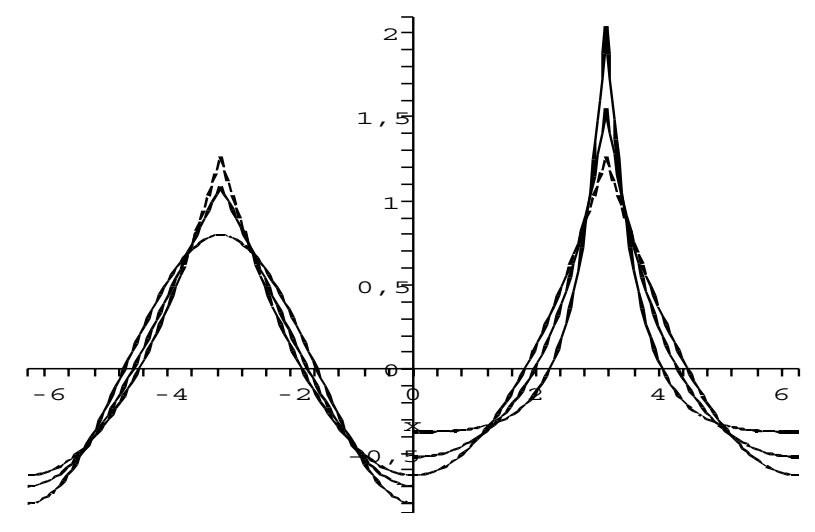

Fig. 1. Plot of one period of the extremal profiles for $L=2 \pi$ and for the normalised value $M=1$. At the left are shown three profiles of increasing amplitude on the trigonometric branch for $q=-1,-2 / 3,0$ ( $q=-1$ corresponds to the smooth harmonic), at the right three profiles with increasing amplitude on the catenary branch for $q=0,1,2$.

Note that by defining the trigonometric branch for $-1<q<0$ (although the sign is irrelevant for the solutions on that branch), we are able to connect the two branches, as we will see. The parameters $a$ and $q$ can be chosen to satisfy the constraint values. On the trigonometric branch the maximal crest height and the steepness at the corner is bounded, while on the catenary branch the maximal crest height and the steepness at the corner monotonically increase to infinity for increasing values of $h / m$. In fact, defining the (half-corner angle) $\phi$ by

$\tan (\phi)=\left[\frac{d \eta}{d x}\right]_{x=\pi}$

we get for the two branches

$\phi_{t r i}=\arctan (a q \sin (q \pi)), \phi_{c a t}=\arctan (a q \sinh (q \pi))$

For $q \rightarrow 0$ the branches singularly meet through a parabolic profile $\eta_{\text {sing }}=a_{0}\left(x^{2}-\pi^{2} / 3\right)$.

Plots of some characteristic profiles on each branch are given in Fig. 1. Plots of the Hamiltonian, of the maximal crest height and of the half-corner angle as function of $q$ are given in Fig. 2.

\section{$4 \mathrm{KdV}$ cnoidal waves and cornered cnoidals}

As we detailed in Sect. 2.5, the periodic nonlinear KdV model, after normalization, leads us to consider the crest problem for the Hamiltonian Eq. (9):

$H(\eta)=\int_{-L / 2}^{L / 2}\left[\frac{1}{2}\left(\partial_{x} \eta\right)^{2}-\frac{1}{3} \eta^{3}\right] d x$

where $L$ denotes the period. First we consider the smooth periodic profiles, the cnoidal wave profiles, after which we

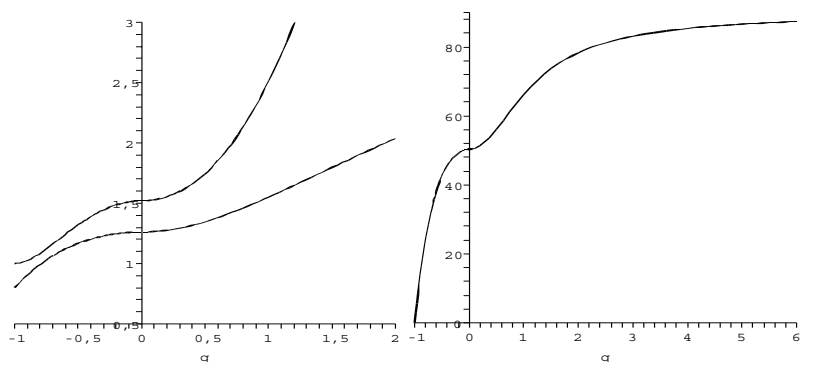

Fig. 2. The left figure shows plots of the Hamiltonian (upper curve) and of the maximal crest height (lower curve) as function of $q$. Here the trigonometric branch is plotted for $-1 \leq q<0$ and the catenary branch for $q>0$. In the same way for the catenary and the trigonometric branch, the half-corner $\phi$ is plotted in degrees in the right figure.

apply the methodology to obtain cornered cnoidal wave profiles.

\subsection{Cnoidal wave profiles}

The normalised equation for relative equilibrium profiles reads

$\delta H=\lambda \delta M+\mu$, i.e. $-\partial_{x}^{2} \eta-\eta^{2}=\lambda \eta+\mu$,

for two multipliers that are such that the momentum constraint is satisfied and that the average of the function vanishes. Periodic functions satisfying Eq. (17) are the so called cnoidal wave profiles; the dynamic solutions are the translation of the profile with fixed speed $\lambda$, which are the well known cnoidal waves, called like this because they can be expressed with Jacobi's elliptic function $\mathrm{CN}$. We now present details of these RE profiles, and of the cornered cnoidal profiles.

Beforehand we note the following expressions for the multipliers. By integrating over a period there results that $\mu$ is related to the momentum like

$\mu=-\int_{-L / 2}^{L / 2} \eta^{2} d x=-2 M(\eta)$

After multiplying the Eq. (17) by $\eta$ and integrating by parts there results

$\lambda=\frac{\int_{-L / 2}^{L / 2}\left[\eta_{x}^{2}-\eta^{3}\right] d x}{\int_{-L / 2}^{L / 2} \eta^{2} d x}$.

The JacobiCN elliptic function is defined for $0 \leq k<1$ by

$c n(z, k)=\cos \phi, z=\int_{0}^{\phi} \frac{d \theta}{\sqrt{1-k^{2} \sin ^{2} \theta}}$

The function $c n$ has periodicity $4 K(k)$ and $c n^{2}$ has periodicity $2 K(k)$, where

$K(k)=\int_{0}^{\pi / 2} \frac{d \theta}{\sqrt{1-k^{2} \sin ^{2} \theta}}$ 


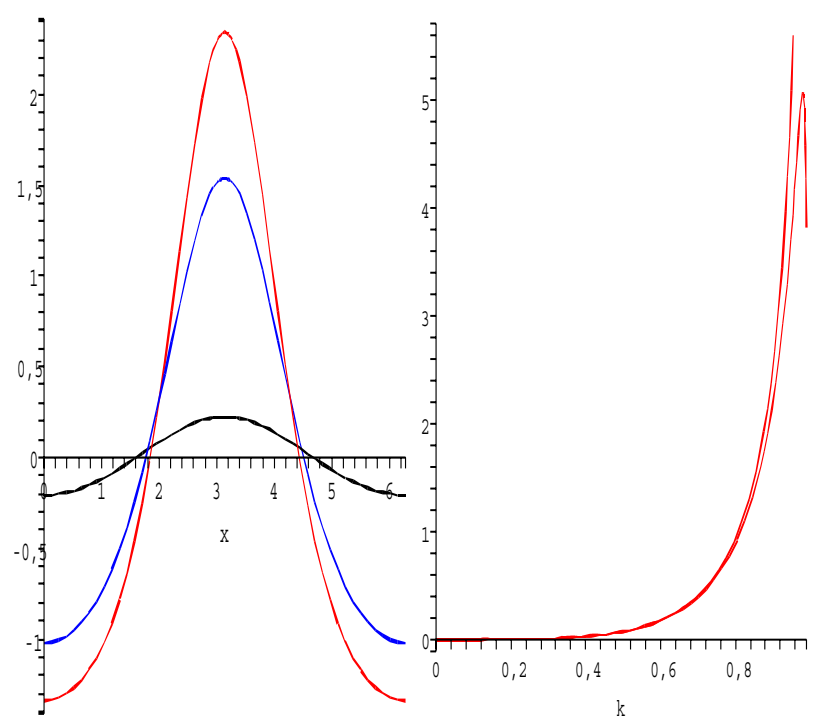

Fig. 3. At the left, plots of the cnoidal wave profiles in one period $L=2 \pi$, for $k=0.5,0.9,0,95$ for increasing amplitude. At the right, plots of momentum (monotonically increasing) and Hamiltonian of the cnoidal waves as function of $k$. Note the non-monotone behavior of the Hamiltonian for $k$ close to 1 .

For $k=0, K(0)=\pi / 2$, while $K(k) \rightarrow \infty$ monotonically for $k \rightarrow 1$. In the following we will use $k$ as a convenient parameter to characterise the profiles; it is in a one-to-one relation with the momentum used in the formulation of the optimization problem.

$\mathrm{KdV}$ cnoidal profiles are then given by

$\eta=6(k \beta)^{2} \cdot c n^{2}\left(\beta\left(x-x_{0}\right), k\right)-b$

where $b$ is such that the average of $\eta$ vanishes, and $x_{0}$ is a shift that makes it possible to get the minimum at $x=0$. The periodicity of the $c n$-function implies that the spatial period of this profile function is $2 K(k) / \beta$. For a given spatial period $\ell$, we have $\beta=2 K(k) / \ell$, and hence we get $\eta=W(x, k ; \ell)-b$ with

$W(x, k ; \ell)=24\left(\frac{k \cdot K(k)}{\ell}\right)^{2} \cdot c n^{2}\left(2 K(k)\left(\frac{x}{\ell}-\frac{1}{2}\right), k\right)$.

This is the expression for profiles for suitable values of $b$ and $k$ for given $\ell$.

Now we fix the value of the period of the periodic solutions we are after to be $L$. In this subsection devoted to cnoidal waves we take $\ell=L$ :

$\eta_{c n}=W(x, k ; L)-\int_{-L / 2}^{L / 2} W(x, k ; L) d x$.

Plots of these (smooth) cnoidal profiles for various values of $k$ are given in Fig. 3 at the left. Observe that for increasing values of $k \rightarrow 1$ the profiles deviate more and more from the

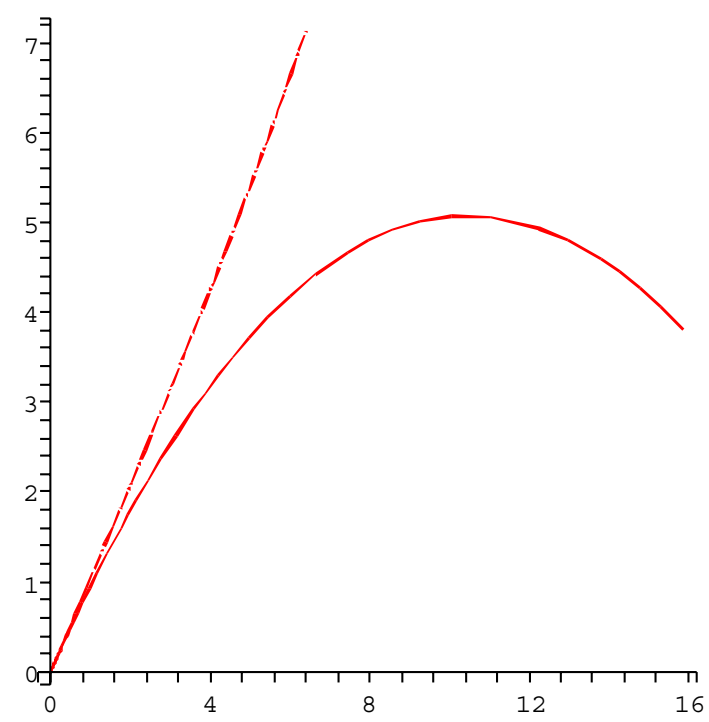

Fig. 4. The plot shows the value function of the cnoidal waves in the momentum (horizontal) - Hamiltonian (vertical) plane. Any cnoidal profile corresponds to a point on this curve, and, visa versa, any other profile will have values of the integrals above this curve. The nearly straight dotted line provides the information for the cornered cnoidal profiles for $q=0.9$.

harmonic function that corresponds to $k=0$. The values of $M$ and $H$ for cnoidal waves as functions of $k$ are also plotted in Fig. 3 at the right. Note that for $k$ close to its limiting value 1 , the value of the Hamiltonian starts to decrease, and tends to $-\infty$ as a consequence of the dominating cubic contribution for large amplitude functions in the Hamiltonian. The RE curve $H=\mathcal{H}(m)$ of $H$ versus $M$ shown in Fig. 4 therefore has a turning point: its derivative, which is equal to the multiplier $\lambda=d \mathcal{H} / d m$, vanishes, changing from positive to negative. Since $\lambda$ is related to the speed of the physical cnoidal wave according to the formula (8), zero crossing of $\lambda$ on this curve, corresponds to increase of the physical velocity above the maximal phase speed $c_{0}$. Obviously, for such too large values of $M$ the $\mathrm{KdV}$-approximation looses its meaning as reliable model.

\subsection{Cornered cnoidal wave profiles}

Employing the methodology explained in Sect. 2, cornered cnoidal (ccn) profiles are obtained for $\ell=L / q$, for $0<q<1$. They are given after adjusting the value of $b$ to guarantee that $\int \eta d x=0$ over one period $[-L / 2, L / 2]$ by

$\eta_{\mathrm{ccn}}=W(x, k ; \ell)-\int_{-L / 2}^{L / 2} W(x, k ; \ell) d x$.

Plots of some characteristic cornered cnoidal profiles are shown in Fig. 5. 


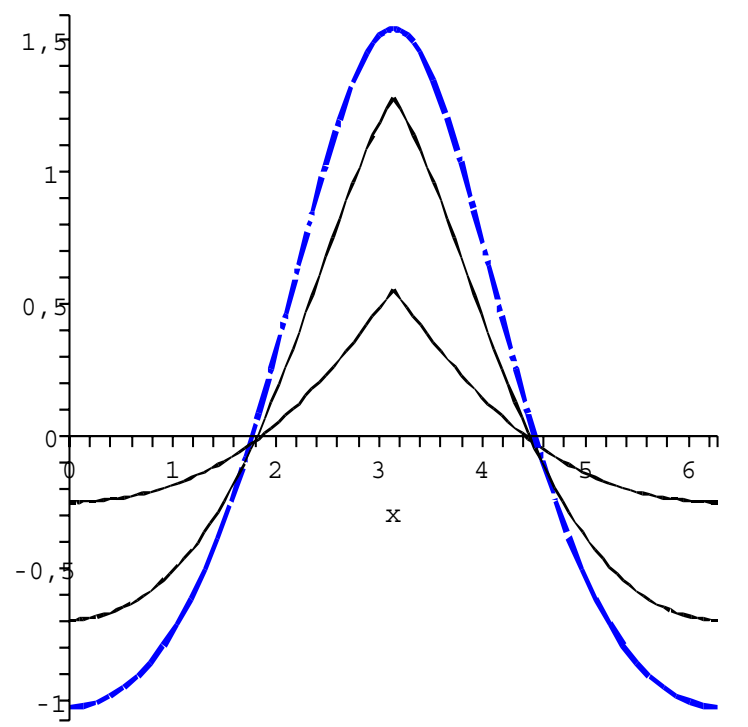

Fig. 5. Extremal profiles are shown in one period $L=2 \pi$ and for the value $k=0.9$. For $q=1$ the smooth cnoidal profile, and for $q=0.9,0.7$ two cornered cnoidals. It must be noticed that for these parameter values all these profiles correspond to different values of $M$ and $H$.

The value of the maximal crest height of the (cornered) cnoidal waves, and of the half corner are shown in Fig. 6 for various values of $q$ as function of $k$. Note that for given $k$ the smooth cnoidal has the largest crest height.

\section{Conclusions}

We have shown that for the KdV model the underlying assumptions described in Sect. 2 of the methodology to obtain extremal periodic wave profiles are satisfied. As a consequence, wave profiles of given period that have maximal crest height are given by the smooth cnoidal profiles when the value of the momentum and energy lie on the boundary of the feasible region. For values of these integrals inside the feasible region, cornered cnoidal profiles are found with corners at the maximal crest. Taking into account the restricted validity of the $\mathrm{KdV}$ model, for not too large values of momentum the results will be realistic. Similar results can be obtained for wave groups modelled by the NLS equation. In Van Groesen and Andonowati (2006a) this was done for finite energy solutions. The methods here can be applied for periodic wave groups. Conversely, the results for periodic KdV profiles can be extended to finite energy profiles of maximal crest height; the KdV solitons are found at the boundary of the feasible region, and cornered solitons in the interior region.

In all the cases mentioned above, the profiles at the boundary of the feasible region are smooth coherent structures.
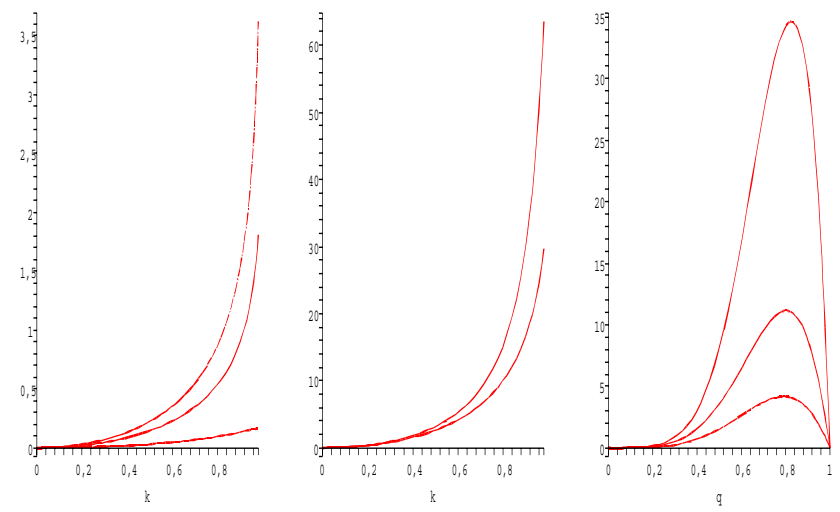

Fig. 6. The plot at the left shows the maximal crest height as function of $k$ for $q=1$ (the smooth cnoidal, dotted), and for cornered cnoidal profiles with $q=0.8$ and $q=0.6$ (lowest). The plot in the middle shows the half corner as function of $k$ for $q=0.6$ and $q=0.9$ (highest). The plot at the right is the half corner as function of $q$ for various values of $k$ : increasing curves correspond to $k=0.5, k=0.7$ and $k=0.9$. For increasing $k$, the value of $q$ for which the sharpest corner is obtained shifts to $q=1$, approaching a vertical tangent (half corner $\pi / 2$ ) for increasing $k$.

These are the Hamiltonian Relative Equilibria: soliton and cnoidal wave (group) profiles. Their dynamics is simple: a translation at constant speed (action of the momentum flow) with the speed determined by the values of the multipliers. Besides that, at this boundary, except from an arbitrary shift, these are the only profiles that are found for these values of the integrals. The situation is very different inside the feasibility region. Then for each pair $(m, h)$ there are infinitely many independent profiles satisfying the integral constraints. Among those the cornered coherent structures have the maximal crest height; their dynamics will be more complicated.

It should be noted that the results obtained here depend strongly on the approximation that is taken for the physical wave energy. Here the usual expression within the KdV model has been used. In a forthcoming paper $?^{3}$ other expressions for the energy (Hamiltonian) of uni-directional waves are derived that include higher order terms; also an expression for the energy of waves on infinite depth are given. Extremal waves for these models will be published elsewhere.

Acknowledgements. E. van Groesen thanks G. Jeurnink and C. van der Meer for useful conversations. This work is part of project TWI.5374 of the Netherlands Organisation of Scientific Research, subdivision Applied Sciences STW.

Edited by: E. Pelinovsky

Reviewed by: two referees

\footnotetext{
${ }^{3}$ van Groesen, E. and Andonowati: Variational derivation of KdV-type of models for surface water waves, Phys. Lett. A., submitted, 2006b.
} 


\section{References}

Olagnon, M. and Prevosto, M. (Eds.): Proceedings Rogue Waves 2004, Ifremer, ISBN 2-84433-150-5, 2004.

Proceedings Rogue Waves: Edinburgh, Scotland, 2005, http://www. icms.org.uk/meetings/2005/roguewaves/index.html, 2005.

van Groesen, E. and de Jager, E. M.: Mathematical structures in continuous dynamical systems, North Holland, Elsevier, Amsterdam, 1994.

van Groesen, E. and Andonowati: Finite energy wave signals of extremal amplitude in the spatial NLS-dynamics, Phys. Lett. A, 357, 86-91, 2006. 